\title{
Impact of inappropriate antifungal therapy according to current susceptibility breakpoints on Candida bloodstream infection mortality, a retrospective analysis
}

\author{
María Fernanda González-Lara', Pedro Torres-González , Patricia Cornejo-Juárez², Consuelo Velázquez-Acosta², \\ Areli Martinez-Gamboa' ${ }^{1}$, Andrea Rangel-Cordero', Miriam Bobadilla-del-Valle', Luis Ostrosky-Zeichner ${ }^{3}$, \\ Alfredo Ponce-de-León ${ }^{1}$ and José Sifuentes-Osornio ${ }^{4^{*}}$
}

\begin{abstract}
Background: The mortality of Candida Bloodstream Infection (CBSI) remains high. Antifungal susceptibility breakpoints were recently updated for Candida species, the impact remains unknown. In this study we evaluated the impact of inappropriate antifungal treatment according to recent breakpoints on 30-day mortality of CBSI.

Methods: From June 2008 to July 2014, data on CBSI episodes from two tertiary-care centers, treated $>72 \mathrm{~h}$ were analyzed. Antifungal therapy and 30-day mortality were registered. Inappropriate antifungal treatment according to current Clinical \& Laboratory Standards Institute (CLSI) breakpoints was adjusted with 30-day mortality-related co-variates.

Results: One hundred forty-nine episodes of CBSI were analyzed. The most frequent species were: C. albicans (40\%), C. tropicalis (23\%) and C. glabrata complex (20\%). According to the 2012 CLSI, 10.7\% received inappropriate treatment. The 30-day mortality was 38\%; severe sepsis [Odds ratio (OR) 3.4; 95\% Cl 1.3-8.4], cirrhosis (OR 36; 95\% Cl 12.2-605), early central venous catheter removal (OR 0.23; 95\% $\mathrm{Cl} 0.08-0.66)$ and previous antifungal therapy (OR $0.15 ; 95 \% \mathrm{Cl} 0.03-0.62)$, were associated with 30-day mortality by multivariate analysis. Inappropriate antifungal treatment was not (OR 0.19; 95\% Cl 0.03-1.2).

Conclusions: Appropriate antifungal therapy according to CLSI 2012 did not have an impact on mortality. Mortality of CBSI remains high due to disease severity and comorbidities; early antifungal therapy and catheter removal may reduce it.
\end{abstract}

Keywords: Antifungal susceptibility testing, Candida bloodstream infections, Fluconazole resistance, Candidemia treatment

\section{Background}

Candida bloodstream infections (CBSI) represent 10\% of all bloodstream infections. The CBSI incidence in North America reaches $0.3-0.9$ cases per 1000 admissions, whereas in Latin America it may reach 5.3 cases per 1000 admissions [1-4]. The mortality rate of CBSI remains high $(46 \%-75 \%)$ despite the availability of effective antifungal therapy [1]. Inappropriate antifungal therapy has been reported as a risk factor for increased

\footnotetext{
*Correspondence: sifuentesosornio@gmail.com

${ }^{4}$ Department of Medicine, Instituto Nacional de Ciencias Médicas y Nutrición Salvador Zubirán, Vasco de Quiroga 15, Belisario Domínguez Sección XVI,

Tlalpan, Zip Code 14080 Mexico City, Mexico

Full list of author information is available at the end of the article
}

mortality [5]. However, routine clinical care in many sites is not guided by antifungal susceptibility testing (AST) but selected according to the species-specific known susceptibility and the patient's clinical condition.

The Clinical and Laboratory Standards Institute (CLSI) recently defined new clinical breakpoints (CBP) for the most common Candida species to categorize them into resistant, intermediate and susceptible and guide antifungal therapy, these breakpoints are now drug and species specific [6]. The rationale for these modifications was the relationship between a higher minimal inhibitory concentration (MIC) and worse outcome showed in some studies, epidemiologic cutoffs values, and pharmacokinetic/pharmacodynamic studies [7]. However, the 
impact of these modifications on mortality remains to be defined. Broth microdilution (BMD) is the reference method for Candida species AST; unfortunately, it is labor intensive, expensive, requires trained personnel, and is subjected to reading bias. Nowadays, antifungal susceptibility testing by automatized systems is considered reliable and may be more easily incorporated into routine clinical care [8-10].

The objective of this study was to assess the impact that the modified antifungal susceptibility breakpoints might have had on 30-day mortality of CBSI.

\section{Methods}

Study design. A retrospective laboratory-based survey was performed; patients admitted to two referral tertiary centers in Mexico City from June 2008 to July 2014 with a positive blood culture for Candida spp., 30-day survival was retrieved. Socio-demographic and clinical variables were recorded in a specially designed case report form. One hundred and forty-nine samples from 149 patients who received antifungal treatment $\geq 72 \mathrm{~h}$ were considered for this analysis. During the study period, AST was not routinely available in the study's centers, the selection of antifungal treatment was decided by the attending physician according to the patient's clinical condition and the Candida species isolated. For this analysis, AST was performed post hoc from the available stored isolates. Our main objective was to identify whether inappropriate antifungal therapy, considering the updated susceptibility breakpoints, was associated with an increased 30-day mortality. A secondary objective was to describe the prevalence of antifungal resistance during the study period.

\section{Definitions}

Empirical therapy was defined as the initiation of an antifungal agent at the first clinical suspicion of fungal infection [11]. Initial antifungal therapy was defined as modification of the empirical antifungal therapy or initiation of any antifungal drug after a positive blood culture with yeasts on a Gram stain, before species identification; whereas definitive treatment was the antifungal administered after species identification. Severe sepsis was defined as the presence of sepsis with any of the following: sepsis-induced hypotension, hyperlactatemia, urine output $<5 \mathrm{ml} / \mathrm{Kg} / \mathrm{h}$ for more than $2 \mathrm{~h}$ following fluid resuscitation, acute lung injury with $\mathrm{PaO}_{2} / \mathrm{FIO}_{2}<250$ or $<200$ in the absence and presence of pneumonia, creatinine $>2 \mathrm{mg} / \mathrm{dL}$, bilirubin $>2 \mathrm{mg} / \mathrm{dL}$, platelet count $<100,000 \mathrm{cell} / \mathrm{mm}^{3}$ or coagulopathy [12].

Antifungal therapy was considered appropriate if the patient received an antifungal agent for which, the Candida isolate was susceptible according to CLSI 2012 breakpoints and inappropriate if the MIC of the isolate was in non-susceptible range $[6,13]$.

\section{Laboratory procedures}

All the Candida spp. isolates recovered from blood cultures during the study period were sent to a central laboratory for identification and AST. The isolates were cultured on Sabouraud media, underwent germ tube testing and were identified using the Vitek 2, (BioMeriéux, Lyon, France). A sample from each culture was stored as water suspension at $-80{ }^{\circ} \mathrm{C}$, until retrieved for susceptibility testing. A small aliquote was unfrozen at room temperature for $24 \mathrm{~h}$, and cultured on Sabouraud agar at $30{ }^{\circ} \mathrm{C}$ for $24 \mathrm{~h}$ to ensure viability. AST was performed on 178 available isolates of which 149 received antifungal treatment and were included for analysis. AST was performed with Vitek 2 using the AST-YS07 card (Biomérieux, Lyon, France) and interpreted according to CLSI guidelines in document M27A3 and its updated version in M27-S4. For those isolates without a species specific clinical breakpoint, epidemiologic cutoff values were used (C. guilliermondii, $C$. pelliculosa and Clavispora lusitaniae). For species without a reported epidemiological cut off value, AST was classified according to the Vitek-2 software (Global CLSI-based + Natural Resistance) (V. 07.01) [14-16].

The reference strains C. parapsilosis ATCC 22019 and C. krusei ATCC 6258 were used as controls. Species identified as C. parapsilosis complex and C. glabrata complex refer to C. parapsilosis complex and C. glabrata complex, since phenotypic testing cannot differentiate the cryptic species within the complexes. Isolates were considered susceptible to echinocandins if the micafungin MIC was within the susceptible ranges, since anidulafungin is not available for YS07 card, and the known variability in the caspofungin MIC with Vitek2 [17, 18]. Assays to identify antifungal resistance mechanisms were not done.

\section{Statistical analysis}

Categorical data was summarized using frequency tables, and the $\chi^{2}$ test was used for comparison between groups. Characteristics of patients were compared using Mann-Whitney $U$ test for continuous variables. To evaluate 30-day mortality in multivariate analysis, we built a multiple logistic regression model including inappropriate antifungal treatment as well as variables with a $p$-value $<0.2$ in univariate analysis or biological plausibility. Stratification for severe sepsis is provided in a separate analysis. Odds ratio (OR) with 95\% confidence interval (CI) was reported. A $p$-value $<0.05$ was considered significant for all the analysis. Stata 11.0 (Stata Corp College Station, TX) software was used for analysis. 


\section{Ethics statement}

The study was reviewed and approved by the Scientific and Bioethics Committee at the Instituto Nacional de Cancerología (reference number INCAN/005/10) and the Research and Bioethics Committee at the Instituto Nacional de Ciencias Médicas y Nutrición "Salvador Zubirán" (reference number 1912). Because of the observational nature of the study, a waiver of informed consent was granted. Authors involved in data analysis could not identify individual patients since the database used numbers specific to the study.

- Availability of data and materials. The datasets used and/or analyzed during the current study are available from the corresponding author on reasonable request.

\section{Results}

During the 72 months of the study period, 83,942 blood cultures were collected in both institutions; $16.2 \%(13,637)$ yielded a positive result, and $1.7 \%$ (227) corresponded to episodes of CBSI. One hundred and seventy-eight isolates (78.4\%) were available for AST at the end of the study period. Of these, $84 \%(149 / 178)$ were episodes treated for $\geq 72 \mathrm{~h}$ and were included for analysis (Fig. 1).

At the time of CBSI diagnosis, 25\% (37/149) of the patients were admitted to intensive care unit (ICU), and $75 \%(112 / 149)$ were admitted to medical or surgical wards. Thirty percent (45/149) had diagnosis of hematologic malignancy and $17.4 \%$ (26/149) other forms of cancer. APACHE II score, to assess mortality and disease severity was available for 122 episodes with a median score of 18.9 (IQR 14-24).

The possible source of the CBSI was identified in $49 \%$ (73/ $149)$ of the episodes, $30.8 \%(46 / 149)$ had an intra-abdominal infection, $7.3 \%$ (11/149) catheter-relatedinfection, and $10.7 \%$ (16/149) had both. Ten percent (16/149) were diagnosed with additional forms of candidiasis (n: 5 hepatosplenic candidiasis, n: 4 skin involvement, n: 3 endocarditis, n: 2 ophthalmic candidiasis, n: 2 osteomyelitis). Fortyfour percent developed severe sepsis, and 22\% (34/149) had neutropenia at CBSI diagnosis of which, $17.6 \%$ (6/34) received primary antifungal prophylaxis. Eighty-two percent $(123 / 149)$ had a central venous catheter in place at the time of CBSI diagnosis. Early central venous catheter (CVC) removal $(<72 \mathrm{~h})$ was achieved in $59 \%(73 / 123)$ of the episodes (Table 1).

\section{Candida species distribution and antifungal susceptibility} Blood cultures yielded a positive result for Candida spp. in a median of 2 days (IQR 1-3) after drawn. The most frequent isolated species were: $C$. albicans (n: 60, 40\%), $C$. tropicalis (n: 34, 23\%), C. glabrata complex (n: 30, 20\%), C. parapsilosis complex (n: 15, 10\%) and C. guilliermondi

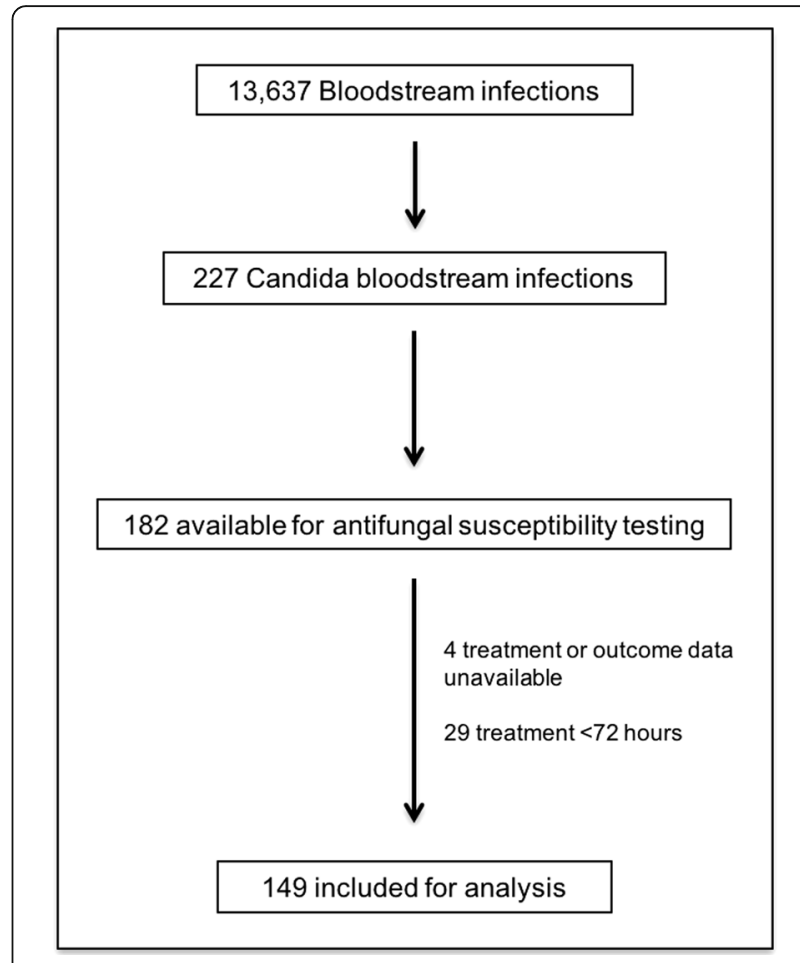

Fig. 1 Flowchart of patients during the study period

(n: 3, 2\%). Other less frequent were: C. krusei (n: 2, 1.3\%), C. norvegensis (n: $1,0.6 \%$ ), C. inconspicua (n: $1,0.6 \%$ ), C. lusitaniae (n:1, 0.6\%) C. lipolytica (n: 1, 0.6\%) and C. pelliculosa (n: 1, 0.6\%).

According to the 2012 CLSI susceptibility breakpoints, $70 \%(104 / 149)$ of the isolates were susceptible to fluconazole, $95.3 \%$ (142/149) to caspofungin and, 97.3\% (145/ $149)$ isolates to micafungin; and $98.6 \%(147 / 149)$ were susceptible to AmB (Table 2).

Overall fluconazole resistance was 6\% (2 C. albicans, 2 C. glabrata complex, 2 C. kruzei, 1 C. parapsilosis complex, 1 C. tropicalis and 1 C. pelliculosa). Overall echinocandin resistance was $1.3 \%$ (1 C. albicans and 1 C. glabrata).

A single echinocandin-resistant C. glabrata was identified, which also was intermediate to fluconazole. One isolate of $C$. albicans showed resistance to every antifungal class (azoles, echinocandins and amphotericin B), from a patient who subsequently developed C. glabrata BSI secondary to a diabetic foot osteomyelitis in a 42-year-old male patient who died after 22 days of treatment with caspofungin.

\section{Antifungal treatment}

Thirty-five of 149 patients (23\%) were receiving antifungal therapy at the time the CBSI was identified, in 23/35 (66\%) cases the indication was empiric therapy for suspected invasive fungal infection, 15/35 (42.8\%) cases were receiving antifungal therapy for another demonstrated 
Table 1 Thirty-day mortality in Candida bloodstream infection. Univariate analysis

\begin{tabular}{|c|c|c|c|c|}
\hline & Total, $n=149$ & 30-d Survivors, $n=93$ & 30-d Non-survivors $n=56$ & $p$-value \\
\hline \multicolumn{5}{|l|}{ Demographics } \\
\hline Female & $78(52.3)$ & $50(53.7)$ & $28(50)$ & 0.65 \\
\hline Age, median (IQR) & $47(32-58)$ & $44(30-55)$ & $48(35.5-61.5)$ & 0.23 \\
\hline Intensive care unit admission & $37(24.8)$ & $15(16.1)$ & $22(39.2)$ & $<0.001$ \\
\hline APACHE $\| n=122$, median (IQR) & $18(14-24)$ & $16(12-20)$ & $23(18.5-28.5)$ & $<0.001$ \\
\hline Severe sepsis & $66(44.3)$ & $29(31.1)$ & $37(66)$ & $<0.001$ \\
\hline \multicolumn{5}{|l|}{ Comorbidities } \\
\hline Hematologic neoplasia & $45(30.2)$ & $22(23.6)$ & $23(41)$ & 0.025 \\
\hline Solid neoplasia & $26(17.4)$ & $17(18.2)$ & $9(16)$ & 0.73 \\
\hline Cardiovascular & $37(24.8)$ & $22(23.6)$ & $15(26.7)$ & 0.7 \\
\hline Chronic kidney disease & $18(12)$ & $10(10.7)$ & $8(14.2)$ & 0.52 \\
\hline Cirrhosis & $11(7.3)$ & $1(1)$ & $10(17.8)$ & $<0.001$ \\
\hline Diabetes mellitus & $19(12.7)$ & $12(12.9)$ & $7(12.5)$ & 0.94 \\
\hline Intra-abdominal infection & $62(41.6)$ & $45(48.3)$ & $17(30.3)$ & 0.031 \\
\hline Neutropenia & $34(22.8)$ & $16(17.2)$ & $18(32.1)$ & 0.035 \\
\hline Cancer $\mathrm{CT}$ & $38(25.5)$ & $18(19.3)$ & $20(35.7)$ & 0.026 \\
\hline CA-BSI & $27(18.1)$ & $22(23.6)$ & $5(8.9)$ & 0.024 \\
\hline CVC & $123(82.5)$ & $80(86)$ & $43(76.7)$ & 0.15 \\
\hline Early CVC removal & $73 / 123(59.3)$ & $55 / 80(68.7)$ & $18 / 43(41.8)$ & 0.004 \\
\hline Total parenteral nutrition & 49 (32.8) & $36(38.7)$ & $13(23.2)$ & 0.034 \\
\hline Empiric antifungal therapy & $35(23.8)$ & $27(29.6)$ & $8(14.3)$ & 0.033 \\
\hline Steroid use & $40(26.8)$ & $20(21.5)$ & $20(35.7)$ & 0.08 \\
\hline Time to antifungal therapy median (IQR) & $2(1-3)$ & $2(1-2)$ & $2(1-3)$ & 0.165 \\
\hline \multicolumn{5}{|l|}{ Initial treatment } \\
\hline Echinocandin & $64(42.9)$ & $42(45.1)$ & $22(39.1)$ & 0.48 \\
\hline Caspofungin & $43(28.8)$ & $27(29)$ & $16(28.5)$ & 0.89 \\
\hline Anidulafungin & $21(14.1)$ & $15(16.1)$ & $6(11.3)$ & 0.4 \\
\hline AmB-d & $61(40.9)$ & 35 (37.6) & $26(46.4)$ & 0.29 \\
\hline Azoles & $25(16.7)$ & $16(17.2)$ & $9(16)$ & 0.85 \\
\hline Fluconazole & $24(16.1)$ & $15(16.1)$ & $9(16)$ & 0.95 \\
\hline Voriconazole & $1(0.6)$ & $1(1)$ & - & - \\
\hline \multicolumn{5}{|l|}{ Definitive treatment } \\
\hline Echinocandin & $56(37.5)$ & $36(38.7)$ & $20(35.7)$ & 0.71 \\
\hline Caspofungin & $31(20.8)$ & $17(18.2)$ & $14(25)$ & 0.34 \\
\hline Anidulafungin & $25(16.7)$ & $19(20.4)$ & $6(10.7)$ & 0.23 \\
\hline AmB-d & $46(30.8)$ & $21(22.5)$ & $25(44.6)$ & 0.005 \\
\hline Azoles & $47(31.5)$ & $36(38.7)$ & $11(19.6)$ & 0.015 \\
\hline Fluconazole & $41(27.5)$ & $31(33.3)$ & $10(17.8)$ & 0.4 \\
\hline Voriconazole & $6(4)$ & $5(5.3)$ & $1(1.8)$ & 0.27 \\
\hline Inappropriate initial antifungal treatment & $13(8.7)$ & $9(9.6)$ & $4(7.1)$ & 0.59 \\
\hline Inappropriate definitive antifungal treatment & $8(5.3)$ & $6(6.4)$ & $2(3.5)$ & 0.36 \\
\hline Inappropriate initial and/or definitive treatment & $16(10.7)$ & $11(11.8)$ & $5(8.9)$ & 0.58 \\
\hline Non-albicans CBSI & $89(59.7)$ & $62(66.6)$ & $27(48.2)$ & 0.026 \\
\hline C. tropicalis & $35(24)$ & $19(20)$ & $16(29)$ & 0.25 \\
\hline
\end{tabular}


Table 1 Thirty-day mortality in Candida bloodstream infection. Univariate analysis (Continued)

\begin{tabular}{lllll}
\hline & Total, $n=149$ & 30 -d Survivors, $n=93$ & 30-d Non-survivors $n=56$ & $p$-value \\
\hline C. glabrata & $28(19)$ & $19(20.4)$ & $9(16)$ & 0.5 \\
C. parapsilosis & $16(10.7)$ & $15(16)$ & $1(1.8)$ & 0.006
\end{tabular}

IQR interquartile range, APACHE acute physiology and chronic health evaluation, $C T$ chemotherapy, CA-BSI catheter associated blood stream infection, CVC central venous catheter, $A m B-d$ amphotericin B-deoxycholate, $C B S I$ candida blood stream infection

Table 2 Antifungal susceptibility of 149 isolates and categorical agreement between CLSI 2008 and 2012

\begin{tabular}{|c|c|c|c|c|c|c|c|c|c|}
\hline \multirow[t]{2}{*}{$\begin{array}{l}\text { Species/ } \\
\text { antifungal (n) }\end{array}$} & \multirow{2}{*}{$\begin{array}{l}\text { MIC range } \\
(\mathrm{mg} / \mathrm{L})\end{array}$} & \multirow{2}{*}{$\begin{array}{l}\mathrm{MIC}_{50} \\
(\mathrm{mg} / \mathrm{L})\end{array}$} & \multirow{2}{*}{$\begin{array}{l}\mathrm{MIC}_{90} \\
(\mathrm{mg} / \mathrm{L})\end{array}$} & \multicolumn{3}{|l|}{$\begin{array}{l}\text { CLSI } 2008 \\
\text { n (\%) }\end{array}$} & \multicolumn{3}{|l|}{$\begin{array}{l}\text { CLSI } 2012 \\
\text { n (\%) }\end{array}$} \\
\hline & & & & $\bar{S}$ & 1 & $\mathrm{R}$ & $\bar{S}$ & $\mathrm{SDD}^{\mathrm{a}} / \mathrm{I}$ & $R$ \\
\hline \multicolumn{10}{|l|}{ C. albicans (60) } \\
\hline Fluconazole & $\leq 1-8$ & 1 & 1 & $60(100)$ & - & - & $56(93.3)$ & $2(3.3)$ & $2(3.3)$ \\
\hline Voriconazole & $\leq 0.12$ & 0.12 & 0.12 & $60(100)$ & - & - & $60(100)$ & - & - \\
\hline Caspofungin & $\leq 0.25-1$ & 0.25 & 0.25 & $60(100)$ & - & - & $56(93.3)$ & $1(1.6)$ & $3(5)$ \\
\hline Micafungin & $\leq 0.06-1$ & 0.06 & 0.06 & $60(100)$ & - & - & $58(96.6)$ & $1(1.6)$ & $1(1.6)$ \\
\hline \multicolumn{10}{|l|}{ C. tropicalis (34) } \\
\hline Fluconazole & $\leq 1-8$ & 1 & 1 & $34(100)$ & - & - & $32(94.1)$ & $1(2.9)$ & $1(2.9)$ \\
\hline Voriconazole & $\leq 0.12$ & 0.12 & 0.12 & $34(100)$ & - & - & $34(100)$ & - & - \\
\hline Caspofungin & $\leq 0.25-1$ & 0.25 & 0.25 & $34(100)$ & - & - & $34(100)$ & - & - \\
\hline Micafungin & $\leq 0.06-0.5$ & 0.06 & 0.06 & $34(100)$ & - & - & $34(100)$ & - & - \\
\hline \multicolumn{10}{|l|}{ C. glabrata (30) } \\
\hline Fluconazole & $\leq 1-64$ & 4 & 8 & $28(93.3)$ & - & $2(6.6)$ & 0 & $28(93.3)$ & $2(6.6)$ \\
\hline Caspofungin & $<0.25-1$ & 0.25 & 0.25 & $30(100)$ & - & - & 29 (96.6) & - & $1(3.3)$ \\
\hline Micafungin & $<0.06-0.5$ & 0.06 & 0.06 & $30(100)$ & - & - & 29 (96.6) & - & $1(3.3)$ \\
\hline \multicolumn{10}{|c|}{ C. parapsilosis (15) } \\
\hline Fluconazole & $\leq 1-8$ & 1 & 8 & $15(100)$ & - & - & $13(86.6)$ & $1(6.6)$ & $1(6.6)$ \\
\hline Voriconazole & $\leq 0.12-0.25$ & 0.12 & 0.12 & $15(100)$ & - & - & $15(100)$ & - & - \\
\hline Caspofungin & $<0.25-1$ & 1 & 1 & $15(100)$ & & & $15(100)$ & - & - \\
\hline Micafungin & $<0.06-1$ & 0.5 & 1 & $15(100)$ & & & $15(100)$ & - & - \\
\hline \multicolumn{10}{|c|}{ C. guilliermondi (3) } \\
\hline Fluconazole & $2-8$ & 2 & 8 & $3(100)$ & & & - & - & - \\
\hline Voriconazole & $<0.12$ & $<0.12$ & $<0.12$ & $3(100)$ & & & - & - & - \\
\hline Caspofungin & $0.25-1$ & 0.25 & 1 & $3(100)$ & & & - & - & - \\
\hline Micafungin & 0.5 & 0.5 & 0.5 & $3(100)$ & & & - & - & - \\
\hline \multicolumn{10}{|l|}{ C. kruzei (2) } \\
\hline Voriconazole & $\leq 0.12$ & 0.12 & 0.12 & $2(100)$ & & & $2(100)$ & - & - \\
\hline Caspofungin & $\leq 0.25-0.5$ & 0.25 & 0.5 & $2(100)$ & & & 1 & 1 & - \\
\hline Micafungin & 0.12 & 0.12 & 0.12 & $2(100)$ & & & 2 & - & - \\
\hline \multicolumn{10}{|l|}{ Other $^{\mathrm{b}}$ (5) } \\
\hline Fluconazole & $\leq 1-16$ & 2 & 16 & $3(60)$ & $2(40)$ & & - & - & - \\
\hline Voriconazole & $\leq 0.12$ & 0.12 & 0.12 & $5(100)$ & - & - & - & - & - \\
\hline Caspofungin & $\leq 0.25->4$ & 0.25 & 4 & $4(80)$ & - & - & - & - & - \\
\hline Micafungin & $<0.06->4$ & 0.12 & 4 & $4(80)$ & - & - & - & - & - \\
\hline
\end{tabular}

MIC minimal inhibitory concentration, $S$ susceptible, $I$ intermediate, $R$ resistant, SDD susceptible dose dependent, CLSI Clinical Laboratory Standards Institute ${ }^{a}$ SDD applies for fluconazole and voriconazole instead of intermediate

${ }^{b}$ Other species: C. Iusitaniae, C. pelliculosa, C. norvegensis, C. lypolitica, C. inconspicua 
fungal infection; $18 / 35$ (51.4\%) patients received azoles (2 voriconazole and 16 fluconazole), 9/35 (25.7\%) received AmB-d and 9/35 (25.7\%) received echinocandins (3 caspofungin and 6 anidulafungin). In 7/35 (20\%) cases, the CBSI agent was non-susceptible to empiric therapy.

Antifungal was initiated or modified within a median of two days (IQR 1-3) after the blood culture was drawn. Echinocandins were the most frequent initial treatment (42.9\%), followed by amphotericin B-deoxycholate (AmBd) $(40.2 \%)$, and fluconazole (16.1\%). One patient received combination therapy with AmB-d and fluconazole, and one received voriconazole. The initial antifungal agent was modified in $37 \%$ (55/149) of the episodes. Among these $47.3 \%(26 / 55)$ were switched to fluconazole after species identification. Twenty-three percent (13/55) were switched to other antifungal due lack of a clinical response; $12.7 \%(7 / 55)$ due to a nonsusceptible isolate (according to the known species-specific susceptibility); $5.4 \%$ $(3 / 55)$ due to toxicity and $5.4 \%(3 / 55)$ due to proven or probable invasive aspergillosis; $3.6 \%(2 / 55)$ to treat other molds and 3.6\% (2/55) due to unspecified reasons. Definitive antifungal treatment of 149 episodes was administered with echinocandins (37.5\%), AmB-d (30.2\%), and fluconazole (31.5\%). One patient received a combination of AmB$\mathrm{d}$ and fluconazole. Among the 89 non-albicans CBSI episodes, 41 (46\%) received echinocandins as definitive treatment, 29 (32\%) AmB-d, and 19 (21.3\%) azoles. The median time of antifungal therapy was 14 days (IQR 5-20).

Regarding the CLSI 2012 breakpoints, 8.7\% (13/149) patients received inappropriate initial antifungal treatment, four of which died: one patient received 12 days of fluconazole $(6 \mathrm{mg} / \mathrm{Kg})$ for a non-susceptible fluconazole $C$. albicans isolate, was switched later to caspofungin due lack of clinical response and died a day after. Two patients received fluconazole for susceptible dose-dependent (SDD) $C$. glabrata; one died five days after CBSI diagnosis, the other was switched to caspofungin on the sixth day and died. The remainder patient had a CBSI due to a fluconazoleresistant $C$. albicans isolate, the patient was started on fluconazole, was switched to caspofungin twelve days later and died 26 days after the CBSI diagnosis. Inappropriate definitive antifungal treatment was administered in 5.3\% (8/ 149) of the episodes (5 C. glabrata, 2 C. albicans, and 1 C. tropicalis), two of which died: one patient received eight days of AmB-d, was switched to fluconazole for a fluconazole -SDD C. glabrata and died 22 days after diagnosis.

No patient received inappropriate initial or definitive treatment according to 2008 guidelines (Table 1).

\section{Outcome}

Thirty-day mortality was 38\% (56/149); non-survivors more often presented with severe sepsis and required mechanical ventilation. Non-survivors were also more likely to have hematologic neoplasia, cirrhosis, severe neutropenia and to receive definitive treatment with AmB-d. Survivors were more likely to have an intraabdominal infection, an early central-venous catheter removal, have received empirical antifungal therapy, to receive definitive treatment with azoles and to have a Non-albicans CBSI (Table 1). In multivariate analysis, severe sepsis (OR 3.4; 95\% CI 1.3-8.4) and previous diagnosis of cirrhosis (OR 36 95\% CI 12.2-605) were independently associated with increased 30-day mortality. Early CVC withdrawal (OR 0.23; 95\% CI 0.08-0.66) and empirical antifungal therapy (OR 0.15 ; $95 \%$ CI $0.03-$ 0.62 ) were independently associated with reduced 30day mortality. When stratified by severe sepsis, survivors were more likely to receive empirical antifungal therapy (OR 0.05; 95\% CI 0.005-0.55) and early CVC removal (OR 0.07; 95\% CI 0.017-0.28) among patients without severe sepsis. Of note neither empirical antifungal therapy nor early CVC removal were associated with reduced mortality in patients with severe sepsis. Inappropriate initial or/and definitive antifungal treatment was not associated with 30 -day mortality (OR 0.19 ; 95\% CI 0.03-1.2), not even after stratification for severe sepsis (Table 3).

Persistent candidemia occurred in 21\% (34/149). Inappropriate treatment was not associated with increased frequency of persistent candidemia $(p=0.7)$.

\section{Discussion}

In this observational study, we did not find an association between inappropriate antifungal therapy defined by the updated CLSI clinical breakpoints and increased mortality. Of note, severe sepsis, and cirrhosis were independently associated with increased mortality. On the contrary, modifiable factors such as empirical antifungal therapy and early CVC removal, were independently associated with decreased 30-day mortality.

The rationale of the CLSI 2012 updates was to identify the emergence of acquired mechanisms of resistance promptly. Recently IDSA treatment guidelines have advocated the testing for azoles to all bloodstream and other relevant isolates for testing, as well as echinocandin testing for those cases with previous exposure and species with known emerging resistance to these drugs, when previous expert recommendations suggested AST only when a patient was not improving $[15,19-21]$. However, it is not clear if AST for CBSI should be routinely performed and the impact of routine AST for CBSI on mortality is not clear $[22,23]$.

In a similar study, in which AST by automatized systems (Sensititre YeastOne) was interpreted according to previous CBP, appropriate early treatment was not associated with survival in multivariate analysis. However, in a subgroup analysis excluding patients who received $<24 \mathrm{~h}$ of appropriate antifungal therapy, the authors 
Table 3 Thirty-day mortality in Candida bloodstream infection. Multivariate analysis

\begin{tabular}{lll}
\hline Characteristic & OR $(95 \% \mathrm{Cl})$ & OR (95\%Cl) without severe sepsis \\
\hline Age & $1.01(0.98-1.03)$ & $1.02(0.99-1.05)$ \\
Inappropriate antifungal treatment $^{\mathrm{a}}$ & $0.16(0.02-1.3)$ & $0.1(0.009-1.05)$ \\
Severe sepsis $_{\text {Cirrhosis }}$ & $3.5(1.4-8.9)$ & - \\
Early CVC withdrawal & $42.1(2.3-744)$ & $56(0.06-47,908)$ \\
Empirical antifungal therapy & $0.22(0.07-0.63)$ & $0.07(0.017-0.28)$ \\
\hline
\end{tabular}

OR Odds ratio; $95 \% \mathrm{Cl}: 95 \%$ confidence interval

${ }^{a}$ According to CLSI 2012, CT Chemotherapy, CVC central venous catheter

found a statistically significant association between survival and appropriate-early treatment [24]. No other study has reported the impact of updated breakpoints on clinical outcome. In this study, we excluded patients with less than $72 \mathrm{~h}$ of antifungal treatment and did not find improved survival among those patients who received appropriate antifungal therapy. However, our definition of appropriateness did not include the dosage of antifungals; this issue becomes more critical while evaluating azoles, which require weight-adjusted loading dose. Contrary to what was expected, the azole therapy showed a tendency to increased survival by univariate analysis, whereas the opposite was observed for AmB-d. This may be explained by the fact that a larger proportion of critical patients received fungicidal treatment rather than fluconazole.

After stratifying for severe sepsis, we identified variables associated with 30-day mortality for CBSI: Cirrhosis, a risk factor for infection and increased mortality due to the multifactorial immune dysfunction occurring in these patients [25-28]. and the removal of a CVC. While several retrospective analysis show increased survival and reduced duration of the CBSI when removing the CVC [29-31]. other studies have not supported these findings [32]. No trial has randomized catheter withdrawal, but a recent patient level quantitative review of 1915 patients from 7 randomized trials confirmed that $\mathrm{CVC}$ removal at any time during treatment was associated with a reduced 30-day mortality, clinical and microbiological succes. In our study, this finding remained significant in both, the C. albicans and the non-albicans Candida subgroups, as well as in the lowest APACHE II score quartiles [33]. Furthermore, empirical antifungal therapy was independently associated with reduced 30day mortality. It has been previously demonstrated that mortality increases wih each subsequent day that empirical therapy is delayed [34, 35].

We found an important proportion of CBSI caused by non-albicans species, the increasing prevalence of nonalbicans CBSI has been previously reported worldwide. $[3,36]$ The increasing rate of isolation of C. glabrata in Brazil and Latin America may be related to older age, geographic factors, and underlying comorbidities such as cancer, particularly in cases not related to selective pressure with fluconazole or areas with low fluconazole resistance rates; $C$. parapsilosis is related to indwelling catheters and chronic conditions and C. tropicalis usually occurs among patients with hematological malignancies, neutropenia and mucositis; all of which are risk factors frequently found in tertiary care centers, as in this study [1].

In this study, most C. albicans isolates remained susceptible to fluconazole and to echinocandins when tested through the automatized system Vitek2. These results agree with a recent report from the SENTRY study using the updated CBPs by BMD [37], and within the range reported by other studies [38-40]. Resistance to fluconazole or echinocandins among $C$. glabrata was an infrequent event, as compared to others [41].

This study has several limitations: This was an observational study thus, there was probable bias in the selection of antifungals by the attending physicians according to disease severity, hindering to predict mortality; the antifungal doses were not systematically registered, which is a sensitive issue regarding azoles; we were unable to retrieve all Candida isolates for performing AST, which was achieved using Vitek 2, instead of the standard of reference broth microdilution (BMD). However, AST by automatized systems is FDA approved and considered to be comparable to BMD, with the benefit of being a standardized, reproducible, easier to perform and less expensive method $[8-10,42]$.

\section{Conclusion}

Inappropriate antifungal treatment according with the current clinical breakpoints was not associated with mortality in this retrospective analysis. Mortality in CBSI remains high due to disease severity and comorbidities such as cirrhosis at the time of diagnosis. In addition, we identified some modifiable factors as early antifungal therapy and catheter removal which may improve the outcome of these patients. Consequently, prospective studies are needed. 


\section{Acknowledgements}

Not applicable.

\section{Funding}

The study was supported by internal funding.

\section{Availability of data and materials}

The datasets used and/or analyzed during the current study are available from the corresponding author on reasonable request.

\section{Authors' contributions}

MFGL contributed to data acquisition, analysis and interpretation and drafting of the article. PTG contributed to conception and design, analysis and interpretation of data, drafting of the article and critical revision for important intellectual content. PCJ contributed to conception and design, analysis and interpretation of data and critical revision for important intellectual content. CVA contributed to data acquisition and critical revision for important intellectual content. AMG contributed to conception and design, analysis and interpretation of data and critical revision for important intellectual content. ARC contributed to data acquisition and critical revision for important intellectual content. MBDV contributed to conception and design, analysis and interpretation of data and critical revision for important intellectual content. LOZ contributed to conception and design, analysis and interpretation of data and critical revision for important intellectual content. APL contributed to conception and design, analysis and interpretation of data and critical revision for important intellectual content. JSO contributed to conception and design, analysis and interpretation of data and critical revision for important intellectual content. All authors read and approved the final manuscript.

\section{Ethics approval and consent to participate}

The study was reviewed and approved by the Research Ethics Committee (Comité de Ética en Investigación) at the Instituto Nacional de Ciencias Médicas y Nutrición "Salvador Zubirán" with the reference number 1912. The Scientific Committee and Bioethics Committee (Comité científico y comité de Bioética) at the Instituto Nacional de Cancerología reviewed and approved the study (Reference number INCAN/CC/005/10). Because of the observational nature of the study, a waiver of informed consent was granted.

\section{Consent for publication}

Not applicable

\section{Competing interests}

J. Sifuentes-Osornio has received research grants from Senosiain, Pfizer, Merck Sharp \& Dhome, Sanofi Pasteur, AstraZeneca, and bioMérieux; personal fees from Pfizer, Merck Sharp\& Dhome, and Sanofi Pasteur; and non-financial support from Pfizer,Merck Sharp \& Dhome, Sanofi Pasteur, and bioMérieux. P. Torres-Gonzalez has received research grants from Pfizer and Sanofi Pasteur. A. Ponce-de-Leon has received research grants from Merck Sharp \& Dhome and Pfizer; and is on the advisory board for Merck Sharp \& Dhome, Pfizer, Janssen-Cilag, and Novartis. L. Ostrosky-Zeichner has received research funding and honoraria from Merck, Astellas, Pfizer, Cidara, T2 Biosystems, and Scynexis. The rest of the authors declare they have no competing interests.

\section{Publisher's Note}

Springer Nature remains neutral with regard to jurisdictional claims in published maps and institutional affiliations.

\footnotetext{
Author details

${ }^{1}$ Department of Infectious Diseases, Instituto Nacional de Ciencias Médicas y Nutrición Salvador Zubirán, Vasco de Quiroga 15, Belisario Domínguez Sección XVI, Tlalpan, Zip Code 14080 Mexico City, Mexico. ${ }^{2}$ Department of Infectious Diseases, Instituto Nacional de Cancerología, Ave. San Fernando 22, Belisario Dominguez Sección XVI, Zip code 14080 Mexico City, Mexico. ${ }^{3}$ University of Texas Health Science Center at Houston, 6431 Fannin. MSB 2.112, Houston, TX 77030, USA. ${ }^{4}$ Department of Medicine, Instituto Nacional de Ciencias Médicas y Nutrición Salvador Zubirán, Vasco de Quiroga 15, Belisario Domínguez Sección XVI, Tlalpan, Zip Code 14080 Mexico City, Mexico.
}

Received: 5 September 2017 Accepted: 21 November 2017 Published online: 06 December 2017

\section{References}

1. Pfaller MA, Diekema DJ. Epidemiology of invasive candidiasis: a persistent public health problem. Clin Microbiol Rev. 2007;20:1.

2. Colombo AL, Nucci M, Park BJ, Nouer SA, Arthington-Skaggs B, da Matta DA, et al. Epidemiology of candidemia in Brazil: a nationwide sentinel surveillance of candidemia in eleven medical centers. J Clin Microbiol. 2006;44:8.

3. Nucci M, Queiroz-Telles F, Alvarado-Matute T, Tiraboschi IN, Cortes J, Zurita $J$, et al. Epidemiology of candidemia in Latin America: a laboratory-based survey. PLoS One. 2013;8:3.

4. Sifuentes-Osornio J, Corzo-Leon DE, Ponce-de-Leon LA. Epidemiology of invasive fungal infections in Latin America. Curr Fungal Infect Rep. 2012;6:1.

5. Parkins MD, Sabuda DM, Elsayed S, Laupland KB. Adequacy of empirical antifungal therapy and effect on outcome among patients with invasive Candida species infections. J Antimicrob Chemother. 2007;60:3.

6. Shah DN, Yau R, Weston J, Lasco TM, Salazar M, Palmer HR, et al. Evaluation of antifungal therapy in patients with candidaemia based on susceptibility testing results: implications for antimicrobial stewardship programmes. J Antimicrob Chemother. 2011;66:9.

7. Fothergill AW, Sutton DA, McCarthy DI, Wiederhold NP. Impact of new antifungal breakpoints on antifungal resistance in Candida species. J Clinical Microbiol. 2014;52:3.

8. Pfaller MA, Diekema DJ, Procop GW, Rinaldi MG. Multicenter comparison of the VITEK 2 yeast susceptibility test with the CLSI broth microdilution reference method for testing fluconazole against Candida spp. J Clin Microbiol. 2007:45:3

9. Peterson JF, Pfaller MA, Diekema DJ, Rinaldi MG, Riebe KM, Ledeboer NA. Multicenter comparison of the Vitek 2 antifungal susceptibility test with the CLSI broth microdilution reference method for testing caspofungin, micafungin, and posaconazole against Candida spp. J Clin Microbiol. 2011;49:5.

10. Pfaller MA, Diekema DJ, Procop GW, Rinaldi MG. Multicenter comparison of the VITEK 2 antifungal susceptibility test with the CLSI broth microdilution reference method for testing amphotericin $B$, flucytosine, and voriconazole against Candida spp. J Clin Microbiol. 2007:45:11.

11. Freifeld AG, Bow EJ, Sepkowitz KA, Boeckh MJ, Ito Jl, Mullen CA, et al. Clinical practice guideline for the use of antimicrobial agents in neutropenic patients with cancer: 2010 update by the infectious diseases society of america. Clin Infect Dis. 2011;52:4.

12. Dellinger RP, Levy MM, Rhodes A, Annane D, Gerlach H, Opal SM, et al. Surviving sepsis campaign: international guidelines for management of severe sepsis and septic shock: 2012. Crit Care Med. 2013;41:2.

13. DePestel DD, Eiland EH, 3rd, Lusardi K, Destache CJ, Mercier RC, McDaneld PM, et al. Assessing appropriateness of antimicrobial therapy: in the eye of the interpreter. Clin Infect Dis. 2014;59;Suppl 3:S154-61.

14. Pfaller MA, Diekema DJ. Progress in antifungal susceptibility testing of Candida spp. by use of clinical and laboratory standards institute broth microdilution methods, 2010 to 2012. J Clin Microbiol. 2012;50:9.

15. Pfaller MA, Andes D, Diekema DJ, Espinel-Ingroff A, Sheehan D. Testing CSfAS. Wild-type MIC distributions, epidemiological cutoff values and species-specific clinical breakpoints for fluconazole and Candida: time for harmonization of CLSI and EUCAST broth microdilution methods. Drug Resist Updat. 2010;13:6.

16. Espinel-Ingroff A, Alvarez-Fernandez M, Canton E, Carver PL, Chen SC, Eschenauer $\mathrm{G}$, et al. Multicenter study of epidemiological cutoff values and detection of resistance in Candida spp. to anidulafungin, caspofungin, and micafungin using the Sensititre YeastOne colorimetric method. Antimicrob Agents Chemother. 2015;59:11.

17. Arendrup MC, Perlin DS. Echinocandin resistance: an emerging clinical problem? Curr Opin Infect Dis. 2014;27:6.

18. Astvad KM, Perlin DS, Johansen HK, Jensen RH, Arendrup MC. Evaluation of caspofungin susceptibility testing by the new Vitek 2 AST-YS06 yeast card using a unique collection of FKS wild-type and hot spot mutant isolates, including the five most common candida species. Antimicrob Agents Chemother. 2013;57:1.

19. Pfaller MA, Diekema DJ, Andes D, Arendrup MC, Brown SD, Lockhart SR, et al. Clinical breakpoints for the echinocandins and Candida revisited: integration of molecular, clinical, and microbiological data to arrive at species-specific interpretive criteria. Drug resistance updat. 2011;14:3. 
20. Pappas PG, Kauffman CA, Andes DR, Clancy CJ, Marr KA, OstroskyZeichner $\mathrm{L}$, et al. Clinical practice guideline for the Management of Candidiasis: 2016 update by the Infectious Diseases Society of America. Clin Infect Dis. 2016;62:4.

21. Nachimuthu N, Ostrosky-Zeichner L. Antifungal susceptibility testing: evolution, indications, and role in clinical practice. Curr Treat Options Infect Dis. 2015:7:3.

22. Oxman DA, Chow JK, Frendl G, Hadley S, Hershkovitz S, Ireland P, et al. Candidaemia associated with decreased in vitro fluconazole susceptibility: is Candida speciation predictive of the susceptibility pattern? J Antimicrob Chemother. 2010;65:7.

23. Ben-Ami R, Rahav G, Elinav H, Kassis I, Shalit I, Gottesman T, et al. Distribution of fluconazole-resistant Candida bloodstream isolates among hospitals and inpatient services in Israel. Clin Microbiol Infect. 2013;19:8.

24. Grim SA, Berger K, Teng C, Gupta S, Layden JE, Janda WM, et al. Timing of susceptibility-based antifungal drug administration in patients with Candida bloodstream infection: correlation with outcomes. J Antimicrob Chemother. 2012;67:3.

25. Chen SY, Tsai CL, Lin CH, Lee CC, Chiang WC, Wang JL, et al. Impact of liver cirrhosis on mortality in patients with community-acquired bacteremia. Diagn Microbiol Infect Dis. 2009;64:2.

26. Bartoletti M, Giannella M, Caraceni P, Domenicali M, Ambretti S, Tedeschi S, et al. Epidemiology and outcomes of bloodstream infection in patients with cirrhosis. J Hepatol. 2014;61:1.

27. Bonnel AR, Bunchorntavakul C, Reddy KR. Immune dysfunction and infections in patients with cirrhosis. Clin Gastroenterol Hepatol. 2011;9:9.

28. Bellot $P$, Frances $R$, Such J. Pathological bacterial translocation in cirrhosis: pathophysiology, diagnosis and clinical implications. Liv Int. 2013;33:1

29. Uzun O, Ascioglu S, Anaissie EJ, Rex JH. Risk factors and predictors of outcome in patients with cancer and breakthrough candidemia. Clin Infect Dis. 2001;32:12.

30. Anaissie EJ, Rex JH, Uzun O, Vartivarian S. Predictors of adverse outcome in cancer patients with candidemia. Am J Med. 1998;104:3.

31. Rex JH, Bennett JE, Sugar AM, Pappas PG, Serody J, Edwards JE, et al. Intravascular catheter exchange and duration of candidemia. NIAID Mycoses Study Group and the Candidemia Study Group. Clin Infect Dis. 1995;21:4

32. Nucci M, Anaissie E, Betts RF, Dupont BF, Wu C, Buell DN, et al. Early removal of central venous catheter in patients with candidemia does not improve outcome: analysis of 842 patients from 2 randomized clinical trials. Clin Infect Dis. 2010;51:3.

33. Andes DR, Safdar N, Baddley JW, Playford G, Reboli AC, Rex JH, et al. Impact of treatment strategy on outcomes in patients with candidemia and other forms of invasive candidiasis: a patient-level quantitative review of randomized trials. Clin Infect Dis. 2012;54:8.

34. Garey KW, Rege M, Pai MP, Mingo DE, Suda KJ, Turpin RS, et al. Time to initiation of fluconazole therapy impacts mortality in patients with candidemia: a multi-institutional study. Clin Infect Dis. 2006:43:1.

35. Kollef M, Micek S, Hampton N, Doherty JA, Kumar A. Septic shock attributed to Candida infection: importance of empiric therapy and source control. Clin Infect Dis. 2012;54:12.

36. Pfaller MA, Diekema DJ, Gibbs DL, Newell VA, Ellis D, Tullio V, et al. Results from the ARTEMIS DISK global antifungal surveillance study, 1997 to 2007: a 10.5-year analysis of susceptibilities of Candida species to fluconazole and voriconazole as determined by CLSI standardized disk diffusion. J Clin Microbiol. 2010;48:4.

37. Pfaller MA, Messer SA, Woosley LN, Jones RN, Castanheira M. Echinocandin and triazole antifungal susceptibility profiles for clinical opportunistic yeast and mold isolates collected from 2010 to 2011: application of new CLSI clinical breakpoints and epidemiological cutoff values for characterization of geographic and temporal trends of antifungal resistance. J Clin Microbiol. 2013;51:8.

38. Marcos-Zambrano LJ, Escribano P, Sanchez C, Munoz P, Bouza E, Guinea J. Antifungal resistance to fluconazole and echinocandins is not emerging in yeast isolates causing fungemia in a Spanish tertiary care center. Antimicrob Agents Chemother. 2014;58:8.

39. Won EJ, Shin JH, Choi MJ, Lee WG, Park YJ, Uh Y, et al. Antifungal susceptibilities of bloodstream isolates of Candida species from nine hospitals in Korea: application of new antifungal breakpoints and relationship to antifungal usage. PLoS One. 2015;10:2.

40. Posteraro B, Spanu T, Fiori B, De Maio F, De Carolis E, Giaquinto A, et al. Antifungal susceptibility profiles of bloodstream yeast isolates by Sensititre YeastOne over nine years at a large Italian teaching hospital. Antimicrob Agents Chemother. 2015;59:7.
41. Vallabhaneni S, Cleveland AA, Farley MM, Harrison LH, Schaffner W, Beldavs ZG, et al. Epidemiology and risk factors for Echinocandin nonsusceptible Candida Glabrata bloodstream infections: data from a large multisite population-based Candidemia surveillance program, 2008-2014. Open Forum Infect Dis. 2015;2:4.

42. Pfaller MA, Diekema DJ, Procop GW, Wiederhold NP. Multicenter evaluation of the new Vitek 2 yeast susceptibility test using new CLSI clinical breakpoints for fluconazole. J Clin Microbiol. 2014;52:6.

\section{Submit your next manuscript to BioMed Central and we will help you at every step:}

- We accept pre-submission inquiries

- Our selector tool helps you to find the most relevant journal

- We provide round the clock customer support

- Convenient online submission

- Thorough peer review

- Inclusion in PubMed and all major indexing services

- Maximum visibility for your research

Submit your manuscript at www.biomedcentral.com/submit
) Biomed Central 